\title{
CARACTERIZAÇÃO ESPECTRAL DA CANA-DE-AÇÚCAR INFECTADA POR NEMATOIDES E MIGDOLUS FRYANUS POR ESPECTRORRADIOMETRIA DE CAMPO
}

\section{In situ spectral characterization of sugar cane infected by nematodes and Migdolus fryanus}

\author{
George Deroco Martins ${ }^{1,2}$ \\ Maria de Lourdes Bueno Trindade Galo ${ }^{1}$ \\ ${ }^{1}$ Programa de Pós Graduação em Ciências Cartográficas-Unesp \\ Faculdade de Ciências e Tecnologia - Presidente Prudente - SP, Brasil. Email:gderoco@yahoo.com.br \\ 2 Universidade Federal de Uberlândia.Email:mlourdes@fct.unesp.br
}

\section{Resumo:}

O cultivo da cana-de-açúcar no Brasil, embora assistido por técnicas modernas de plantio, é alvo constante de parasitas do sistema radicular. Por registrar seletivamente o fluxo espectral da radiação eletromagnética refletida pela vegetação, o sensoriamento remoto tornou-se uma poderosa ferramenta na detecção das plantas infectadas por patógenos do solo. Com o objetivo de caracterizar espectralmente a cana-de-açúcar sadia e infectada por nematoides e pela larva do besouro Migdolus fryanus, foram tomadas medidas radiométricas in situ e geradas curvas hiperespectrais de plantas sadias e infectadas. Técnicas específicas de análise espectral, como a determinação da posição da borda do vermelho limítrofe (Red Edge Position Determination REPD) e diferentes índices espectrais foram avaliados para discriminar as três ocorrências. As curvas de reflectância mostraram diferenças em magnitude principalmente nos comprimentos de onda do vermelho e infravermelho próximo e, assim como a determinação do REP e os índices de clorofila $b$, NDVI, MCARI e TCARI, permitiram distinguir apenas entre plantas sadias e infectadas. As razões espectrais sensíveis aos pigmentos clorofila $a$ e carotenoides, porém, discriminaram as três ocorrências, inclusive plantas infectadas por nematoides e Migdolus fryanus. A melhor discriminação foi obtida com o índice de carotenoides, um pigmento fortemente relacionado com estresse da planta.

Palavras-chave: Sensoriamento remoto hiperespectral, pragas da cana-de-açúcar, patógenos radiculares, análise espectral, índice de vegetação.

\begin{abstract}
:
Despite all technological support used in sugarcane cultivation in Brazil, this planting is still subject to the action of root pathogens. Due to its ability to selectively record the spectral flux of electromagnetic radiation reflected by vegetation, remote sensing is a powerful tool in detecting plants infected by pests and parasites. The objective of this work was to characterize the spectral response of healthy sugarcane and its plants infected by nematodes and Migdolus fryanus beetle larva. In situ radiometric measurements were performed and specific spectral analysis techniques, such as Red Edge Position Determination (REPD) and different vegetation indices were evaluated to discriminate these occurrences. The reflectance curves generated showed differences in magnitude mainly at red and near-infrared wavelengths and, as well as REPD,
\end{abstract}


chlorophyll $b$ index, NDVI, MCARI and TCARI, were able to discriminate only healthy and infected plants. The spectral ratios sensitive to chlorophyll $a$ and carotenoids pigments, however, discriminated the three occurrences, including nematode-infected plants and Migdolus fryanus. The best discrimination was obtained by the index of carotenoids, a pigment whose content is related to plant stress.

Keywords: hyperspectral remote sensing, sugarcane pests, root pathogens, spectral analysis, vegetation index.

\section{Introdução}

Como ocorre com outras culturas agrícolas de importância econômica no Brasil, a cana-deaçúcar está sujeita a ação de pragas e parasitas. A ocorrência de áreas canavieiras infectadas se constitui não apenas em fator limitante à produtividade agrícola, mas também causa problemas de ordem ecotoxicológica decorrentes das práticas de controle que se apoiam na aplicação indiscriminada de agrotóxicos (Corbani, 2008). Especificamente na região oeste do estado de São Paulo, os agentes que mais contribuem para a queda da produtividade canavieira são os parasitas e pragas do solo, como nematoides e a larva do besouro Migdolus fryanus.

Ambos, parasita e praga, são encontrados em diversos tipos de solos, porém causam maiores danos em lavouras cultivadas em solos arenosos. O início do parasitismo é caracterizado pelo ataque ao sistema radicular da planta, prejudicando consideravelmente o desenvolvimento e o crescimento do vegetal. No caso de infecções severas pode ocorrer a morte prematura de parte da cultura agrícola (Omarjee et al., 2008).

O monitoramento e o gerenciamento da cana-de-açúcar são essenciais para avaliar a sustentabilidade da produção de açúcar e etanol no Brasil e, da mesma forma que há necessidade de mapear e quantificar a produtividade da cultura canavieira é preciso detectar áreas cultivadas infectadas por pragas e parasitas. Pelo seu caráter sinóptico e repetitivo, o Sensoriamento Remoto tornou-se ferramenta importante no monitoramento agrícola, principalmente por permitir o acompanhamento da cultura ao longo do seu ciclo de desenvolvimento, viabilizar estimativas de safra e a determinação tanto de novas áreas de cultivo quanto da produtividade agrícola (Rudorff, 2010). Além disso, essa tecnologia é uma alternativa consistente na detecção da cana-de-açúcar infestada por parasitas e pragas, principalmente por oferecer a possibilidade de registrar as variações espectrais associadas à mudança nas condições fitopatológicas da cultura.

Como ocorre para a vegetação em geral, a resposta espectral das culturas agrícolas depende de uma série de fatores bioquímicos do vegetal, além das características físicas do dossel. Normalmente, esses fatores estão condicionados a arquitetura do dossel, química foliar, parâmetros agronômicos e condições atmosféricas (Abdel-Rahman et al., 2008). Para alguns autores que concentraram esforços no estudo da cana-de-açucar (Apan et al., 2004; Galvão et al., 2006; Rao, 2008; Bégué et al., 2010; Gonçalves et al., 2012), o fator que mais afeta as propriedades ópticas da cultura é a estrutura geométrica da parte superior do dossel.

Além disso, essa resposta espectral também é influenciada pelos pigmentos presentes na folha, tais como a clorofila $a$ e $b$, carotenos, xantofila e antocianinas (Abdel-Rahman et al., 2008), que provocam a absorção da radiação eletromagnética (REM) ou influenciam nos processos fisiológicos associados ao desenvolvimento do vegetal. Além dos pigmentos, a deficiência de nitrogênio foliar altera consideravelmente a reflectância do vegetal ao longo de todo intervalo 
espectral (Grisham, et al. 2010), enquanto o conteúdo de água nas folhas define bandas de absorção em comprimentos de ondas específicos (980 nm e $1205 \mathrm{~nm}$ ) (Galvão et al. 2005).

Em geral, o padrão espectral da vegetação sadia na região do visível é caracterizado por duas bandas de absorção bem definidas, explicadas pela ação da clorofila, no intervalo espectral do azul e do vermelho, entre $450 \mathrm{~nm}$ e $520 \mathrm{~nm}$ e entre $630 \mathrm{~nm}$ e $690 \mathrm{~nm}$, respectivamente. Já na faixa do infravermelho, a reflectância é alta e aumenta gradativamente com o acréscimo da quantidade de folhas, devido às múltiplas reflexões da radiação incidente nos espaços intracelulares do mesófilo foliar. Esse comportamento padrão da reflectância sofre variações quando a planta está em senescência ou em condições de estresse ambiental. A ausência de clorofila provoca redução na quantidade de energia na região espectral do visível, com maior reflectância no verde e no vermelho, passando a ter aparência amarelada ou clorótica (Carter, 1993). Os valores maiores de reflectância em torno de $700 \mathrm{~nm}$ representam o que é frequentemente chamado de "deslocamento da borda vermelha para o azul" (blue shift of the red edge), com referência ao deslocamento do gradiente da transição entre o vermelho e o infravermelho próximo na direção aos menores comprimentos de onda, perceptível na curva da resposta espectral da vegetação.

A possibilidade de detectar mudanças estruturais ou fisiológicas na vegetação envolve a aquisição minuciosa e detalhada de medidas da reflectância em intervalos espectrais mais estreitos. Nesse sentido, os espectrorradiômetros que realizam coletas radiométricas in situ podem fornecer não apenas dados detalhados sobre as características espectrais dos alvos, como também permitem adquirir valores físicos, como a radiância e a reflectância, que caracterizam espectralmente diferentes objetos.

Diante do exposto e considerando a dificuldade em diagnosticar antecipadamente a ocorrência tanto de nematoides, quanto da larva do besouro na cana-de-açúcar, agravado pelo fato de que ambos se instalam no solo e no sistema radicular, entende-se que a detecção precoce e a discriminação desses tipos de infecção poderiam conduzir práticas de controle e manejo específicas para cada ocorrência. Nesse contexto, o objetivo desta pesquisa é caracterizar espectralmente a cana-de-açúcar sadia e a infectada por nematoides e Migdolus fryanus, a partir de medidas espectrorradiométricas tomadas in situ e análise das curvas espectrais, avaliando o potencial da determinação da posição da borda do vermelho (Red Edge Position Determination REPD) e de diferentes índices hiperespectrais sensíveis à variação de clorofila na discriminação entre a cultura sadia e infectada.

\section{MATERIAL E MÉTODOS}

O presente estudo envolveu o desenvolvimento de um experimento em áreas de cultivo de canade-açúcar da usina sucroalcooleira UMOE Bioenergy de Sandovalina, município localizado na região oeste do estado de São Paulo. A fim de verificar as condições locais quanto à ocorrência de nematoides e Migdolus fryanus, foram realizados levantamentos exploratórios in situ, durante o ciclo de desenvolvimento da cultura e registrados localmente a presença da praga e do parasita. No período compreendido entre julho e outubro de 2012 foram inspecionadas imagens orbitais do sistema RapidEye da área, com o intuito de caracterizar o padrão de ocorrência das áreas infestadas (Figura 1) e identificar talhões com aproximadamente 12 meses do plantio, com canade-açúcar nas três condições de estudo: sadia e infectada pela praga e parasita. Retornando ao campo, foram definidos elementos amostrais, com o suporte dos técnicos da usina, para posterior aquisição das medidas em cada uma das ocorrências. 


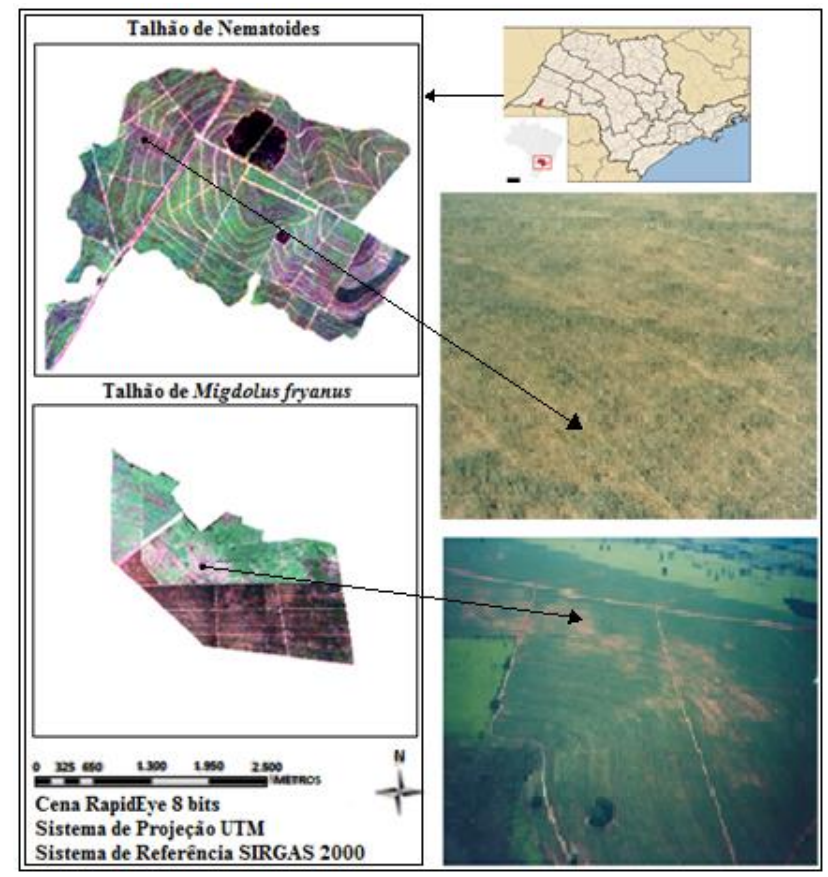

Figura 1: Localização geográfica das áreas experimentais no Estado de São Paulo. Em destaque, composições coloridas RGB de imagem RapidEye indicando talhões de cana-de-açúcar infectados, associados a imagens áreas que caracterizam a ocorrência em campo de nematoides (extraída de www.interural.com) e M. fryanus (www.cana.com.br).

As medições espectrorradiométricas foram feitas nos dias 15 e 18 de outubro de 2012, entre 11:00 e 14:00horas, em dez (10) elementos amostrais previamente definidos para cada uma das ocorrências (plantas sadias, nematoides e Migdolus fryanus). O instrumento de medição foi um espectrorradiômetro portátil ASD FieldSpec, modelo HandHeld UV/VNIR, que opera no intervalo espectral entre 375 e $1075 \mathrm{~nm}$, configurado em 512 canais com 1,6 nm de resolução espectral.

Durante a aquisição das medidas de radiância do alvo e do fluxo retroespalhado, o sensor foi posicionado a uma altura aproximada de $50 \mathrm{~cm}$ acima do topo do dossel da vegetação, sustentado por um tripé e um bastão, camuflados por uma cobertura de tecido preta para evitar a influência da resposta espectral do suporte. Restringiu-se o campo de visada do sensor a $10^{\circ}$, com o intuito de minimizar a interferência de alvos vizinhos, o que definiu um GSD (Ground Sample Distance) de $8,75 \mathrm{~cm}$. Para cada elemento amostral utilizou-se a média de dez (10) repetições da leitura de radiância do alvo e mediu-se também a radiância de uma superfície lambertiana de referência (placa Spectralon), nas mesmas condições de iluminação e de observação, para estimar o Fator de Reflectância Hemisférico Cônico (FRHC) do alvo.

Rigorosamente, define-se o Fator de Reflectância Bidirecional (FRB) pela razão entre o fluxo radiante do alvo pelo fluxo que seria refletido por uma superfície Lambertiana, sem perdas por absorção e transmissão, e sob as mesmas condições de geometria de iluminação e visada (Jensen, 2009). Em condições de campo, quando a placa de referência passa a ser um difusor não perfeito e para pequenos ângulos de visada (menores que $20^{\circ}$ ) o FRB é descrito pela Equação 1 . 


$$
\operatorname{FRB}\left(\omega_{\mathrm{i}} \omega_{\mathrm{r}}\right)=\frac{\mathrm{dL}\left(\theta_{\mathrm{r}}, \Phi_{\mathrm{r}}\right)(\text { alvo })}{\mathrm{dL}\left(\theta_{\mathrm{r}}, \Phi_{\mathrm{r}}\right)(\text { referência })} \mathrm{K}\left(\theta_{\mathrm{i}}, \Phi_{\mathrm{i}}, \theta_{\mathrm{r}}, \Phi_{\mathrm{r}}\right)
$$

em que: $d L$ é radiância refletida (infinitesimal) pelo alvo ou placa, $\omega_{r}$ é o ângulo sólido, $\theta$ e $\Phi$ remetem ao ângulo zenital e o azimutal, respectivamente, $i$ está associado ao fluxo incidente e $r$ ao refletido, enquanto $K$ é o fator de correção da placa.

Embora o FRB seja majoritariamente considerado como a componente física da radiação eletromagnética mensurada em campo e laboratório, autores como Martonchik et al., (2009); Milton et al., (2009), Schaepman-Strub et al., (2006), entre outros, argumentam que se a geometria das medidas em campo for mantida constante (mesma iluminação ambiente e campo de visada instrumental), a componente medida é o Fator de Reflectância Hemisférico Cônico (FRHC). Assim, levando-se em conta as condições em que foram tomadas as medidas in situ, adotou-se essa terminologia neste artigo.

A partir da conversão dos valores de Radiância em FRHC, utilizou-se a média das dez repetições por ponto para produzir uma única curva por elemento amostral. Em seguida, foi aplicado um filtro média móvel de 3 pontos para suavizar as curvas, minimizando o efeito de ruídos causados por interferências externas ao alvo e pela imprecisão do instrumento.

Tratamentos específicos foram aplicados às curvas de FRHC, tais como a determinação da posição da borda do vermelho limítrofe (REPD - Red Edge Position Determination) (Apan et al. 2004; Cho et al., 2006) e o cálculo de índices hiperespectrais (Dawghtry et al., 2000; Haboudane et al., 2002; Liu et al. 2010), normalmente usados para inferir algumas características biofísicas da vegetação, inclusive associadas às sua condições fitossanitárias.

Segundo Jensen (2009) o intervalo espectral entre 680 e 800nm, denominado Borda do Vermelho (Red Edge, RE), é caracterizado pela mudança abrupta do comportamento na curva espectral de um vegetal, decorrente de uma série de fatores relacionados à absorção pela clorofila e espalhamento interno da Radiação Eletromagnética (REM). Nesse sentido, a REPD é uma técnica usada para avaliar o comportamento da borda do vermelho, já que essa posição (REP) está intrinsecamente relacionada com as taxas de nitrogênio e clorofila foliar (Ju et al., 2010). Várias técnicas foram desenvolvidas para avaliar a REP, porém a solução proposta por Cho et al. (2006), denominada Método da Extrapolação Linear, foi adotada neste trabalho. Tal método, que consiste em determinar a REP por meio da intersecção de dois polinômios de primeiro grau, tangenciados sobre os pontos de inflexão da curva espectral resultante da primeira derivada dos valores de FRHC no intervalo correspondente ao vermelho limítrofe, foi aplicado às curvas espectrais obtidas para cada uma das três ocorrências.

Os índices hiperespectrais calculados a partir das curvas de FRHC em cada um dos dez pontos de coleta para cada condição avaliada foram: razões simples sensíveis à quantidade de clorofila $a$, clorofila $b$ e carotenoides na vegetação (Blackburn, 1998), o índice de vegetação da diferença normalizada (NDVI) e as razões que relacionam a profundidade das bandas de absorção na região espectral do visível com os níveis de clorofila foliar (Dawghtry et al., 2000; Haboudane et al., 2002). A utilização de índices na discriminação espectral da vegetação sadia, infectada por nematoides e Migdolus fryanus deve-se à sua característica de minimizar, entre outros, o efeito de variações nas condições de iluminação da cena (Ponzoni et al., 2015).

Os pigmentos considerados na formulação das razões espectrais são aqueles sensíveis a quantidade de clorofila $a$ (Equação 2), clorofila $b$ (Equação 3) e carotenoides (Equação 4). Nessas formulações $R_{\lambda}$ representa o FRHC para um comprimento de onda específico $(\lambda)$, expresso em nm: 


$$
\begin{gathered}
\text { Clorofila } a=\mathrm{R}_{800} / \mathrm{R}_{670} \\
\text { Clorofila } b=\mathrm{R}_{800} / \mathrm{R}_{635} \\
\text { Carotenóides }=\mathrm{R}_{800} / \mathrm{R}_{470}
\end{gathered}
$$

O NDVI (Normalized Difference Vegetation Index) tem sido utilizado na geração de perfis sazonais e temporais que permitem avaliar o estresse da vegetação (Rouse et al., 1973) e é obtido pela Equação 5:

$$
\text { NDVI }=\left(R_{864}-R_{671}\right) /\left(R_{864}+R_{671}\right)
$$

Os indicadores de clorofila foliar considerados neste estudo são adaptações do índice Chlorophyll Absorption Ratio Index (CARI). O MCARI (Modified Chlorophyll Absorption Ratio Index), proposto por Dawghtry et al. (2000), avalia a profundidade da banda de absorção da clorofila em $670 \mathrm{~nm}$ por meio de uma relação matemática formulada a partir de valores de reflectância obtidos em 550nm e 700nm (Equação 6). Já o TCARI (Transfomed Chlorophyll Absorption Ratio Index) desenvolvido por Haubodane et al. (2002) e indicado na Equação 7, procura compensar as variações espectrais características da influência dos materiais de fundo, considerando os mesmos comprimentos de onda.

$$
\begin{gathered}
\text { MCARI }=\left[\left(\mathrm{R}_{700}-\mathrm{R}_{670}\right)-0,2\left(\mathrm{R}_{700}-\mathrm{R}_{550}\right)\right]\left(\mathrm{R}_{700} / \mathrm{R}_{670}\right) \\
\text { TCARI }=3\left[\left(\mathrm{R}_{700}-\mathrm{R}_{670}\right)-0,2\left(\mathrm{R}_{700}-\mathrm{R}_{550}\right)\left(\mathrm{R}_{700} / \mathrm{R}_{670}\right)\right]
\end{gathered}
$$

As respostas espectrais obtida para cana-de-açúcar sadia e infectada por nematoides e Migdolus fryanus foram analisados por meio das curvas de FRHC, das REP e dos índices hiperespectrais, considerando os valores médios e respectivos desvios padrão. Para avaliar graficamente o potencial de discriminação desses indicadores espectrais, foram utilizados os valores médios e intervalos de variação dos índices para construir boxplots para cada uma das três ocorrências.

\section{RESULTADOS E DISCUSSÃO}

As curvas de FRHC suavizadas por filtro média de três pontos, obtidas para cada elemento amostral, são mostradas na Figura 2-A. Todas essas curvas, independente de terem sido geradas a partir de medidas na cana-de-açucar sadia (curvas em preto), infectada por nematoides (vermelhas) ou por Migdolus fryanus (verdes) mostram as feições características de absorção e espalhamento típicas da reflectância da vegetação, mas com perceptíveis variações em magnitude nas regiões do visível e infravermelho próximo. As curvas médias, elaboradas para 
cada uma das ocorrências (Figura 2-B), indicam as diferenças entre a vegetação sadia e a infectada por nematoides e Migdolus fryanus.

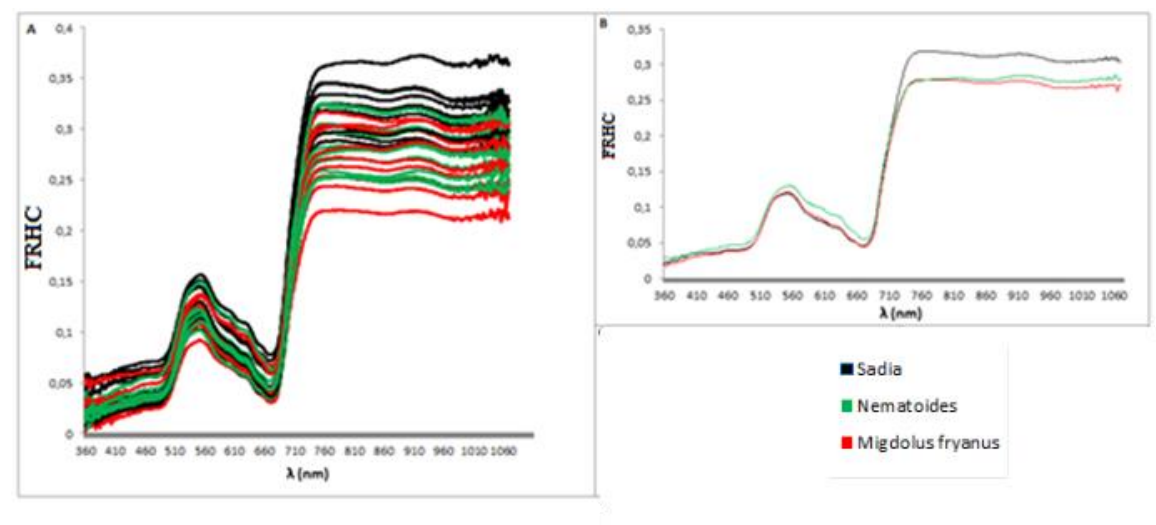

Figura 2: Curvas espectrais do FRHC. Espectros obtidos para cada um dos elementos amostrais medidos in situ, no intervalo espectral de 360 a 1050nm (A), curvas médias de cada uma das ocorrências estudadas (B).

A configuração média das curvas, ao longo do espectro visível (Figura 2-B), indica que a canade-açúcar parasitada por nematoides e as plantas sadias apresentaram as bandas de absorção clássicas das clorofilas $a$ e $b$, o que não ocorreu para a cana-de-açúcar infectada pela larva do besouro Migdolus fryanus, que manteve valores médios de FRHC maiores no vermelho (600 a $680 \mathrm{~nm}$ ). Esse comportamento espectral é consistente para uma cultura infectada pela larva do besouro, devido ao comprometimento da estrutura foliar do vegetal, que torna as folhas cloróticas e amareladas (Perin et al., 2006).

Na região do infravermelho próximo (700 a 1050nm) é marcante a diferença no comportamento espectral médio da vegetação sadia e infectada. As médias calculadas para a cana-de-açúcar infectada são similares para as duas ocorrências até aproximadamente o 800nm. A partir desse comprimento de onda, a resposta espectral da vegetação parasitada por nematoides passa a apresentar aumento sutil nos valores de FRHC quando comparada à cana-de-açúcar infectada por Migdolus fryanus.

Para mostrar que a influencia das propriedades espectrais das plantas sadias e infectadas determinam as diferenças no FRHC, apresenta-se, na Figura 3, um gráfico que relaciona essa medida com as variações no ângulo zenital do sol, registradas durante o intervalo de tempo de tempo em que as medidas foram adquiridas, nos comprimentos de onda de $671 \mathrm{~nm}$ e $864 \mathrm{~nm}$. 


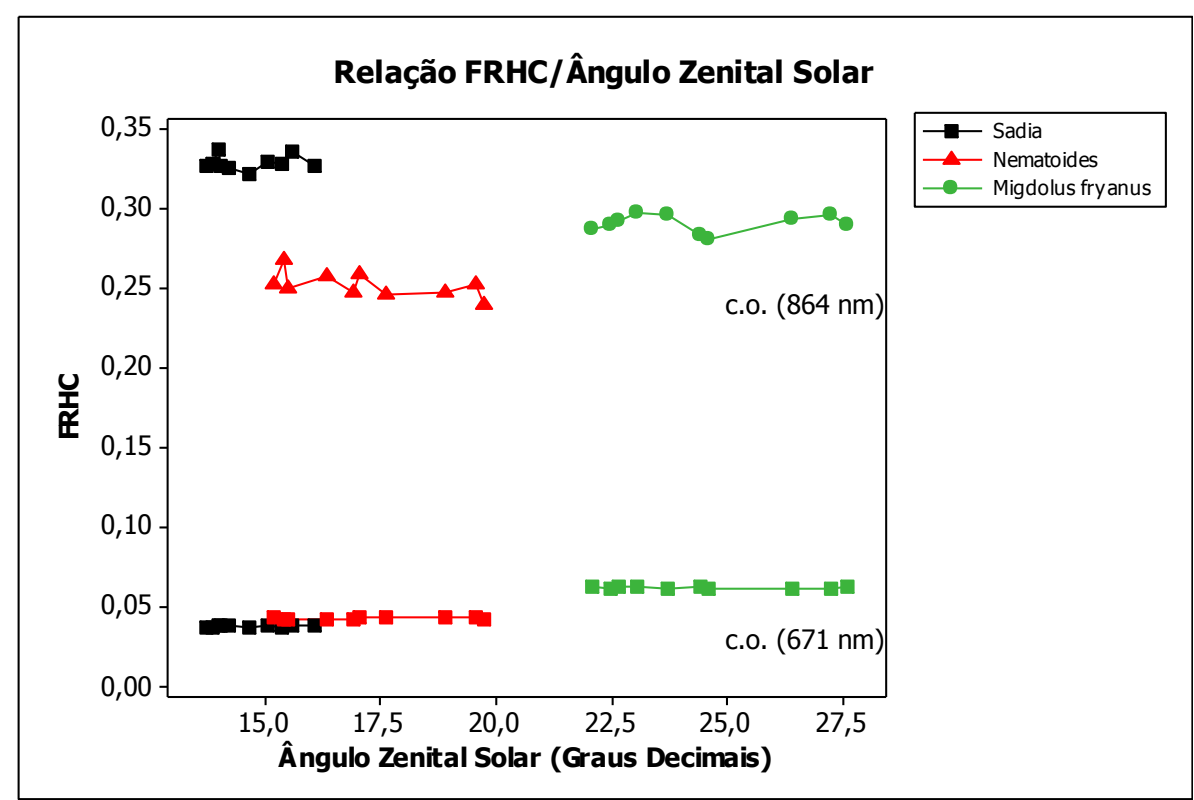

Figura 3: Relação entre o FRHC medido entre as variações no Ângulo Zenital Solar para os comprimentos de onda de 671 e $864 \mathrm{~nm}$.

A exemplo do que é relatado por Kimes et al. (1979), observou-se que os dosséis planófilos foram poucos influenciados pela variação do ângulo zenital solar, de modo que as medidas tomadas da vegetação sadia e infectada por nematoides, sob mesmo ângulo zenital da vegetação, resultaram em pequena variação no comprimento de onda do vermelho $(671 \mathrm{~nm})$, mas uma variação significativa de FRHC no IVP $(864 \mathrm{~nm})$. O decréscimo no FRHC registrado para Migdolus fryanus em $864 \mathrm{~nm}$ ainda poderia ser atribuído as maiores ângulos zenitais do sol, porém o pequeno acréscimo observado em $671 \mathrm{~nm}$ descaracteriza a influência desse ângulo relacionado com o horário de aquisição de medidas.

As diferenças observadas no FRHC nos comprimentos de onda expressos na Figura 3 sugerem uma possível discriminação dessas ocorrências pelo REP. A determinação da posição da borda do vermelho limítrofe pelo método proposto por Cho et al. (2006) resultou em comprimentos de onda distintos (entre 700 a $723 \mathrm{~nm}$ ) para plantas infectadas e cana-de-açúcar sadia, porém a esperada separação entre os dois tipos de infecção não se concretizou, como indica a sobreposição observada nos comprimentos de onda da REP para as amostras de cana-de-açúcar infectada por nematoides e Migdolus fryanus, expressa no gráfico de barras da Figura 4, o qual relaciona a frequência de observações com uma mesma posição de borda do vermelho limítrofe (REP), discriminadas por tipo de ocorrência. 


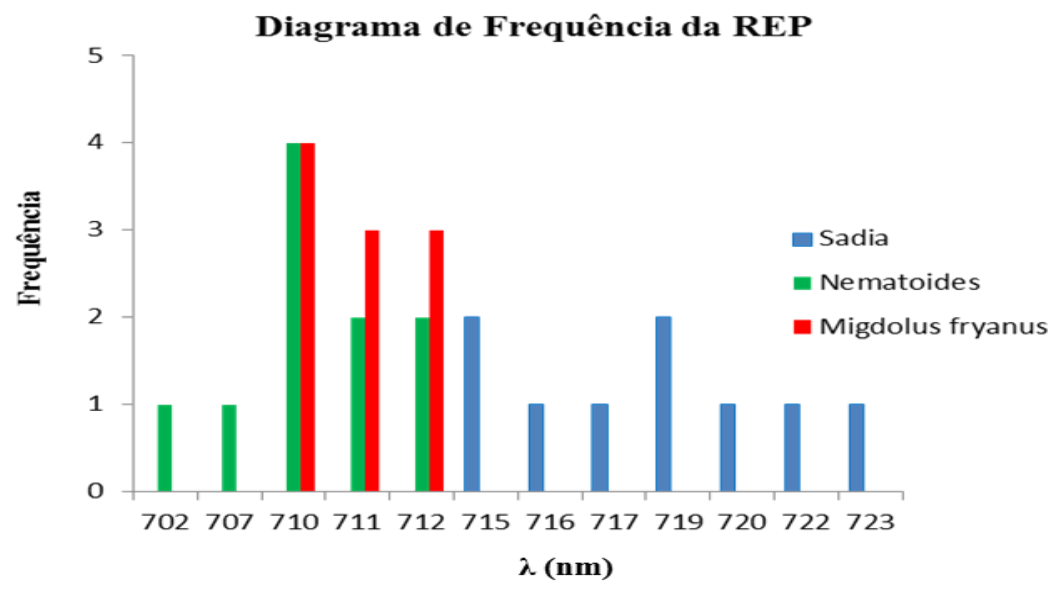

Figura 4: Frequência de ocorrência dos valores de REP para elementos amostrais associados às categorias sadia, infectada por nematoides e por Mygdolus fryanus.

Pela Figura 4 verifica-se que os valores da REP para cana-de-açúcar sadia estão na região espectral do infravermelho próximo (entre 710 e $723 \mathrm{~nm}$ ), enquanto que para plantas infectadas pelo parasita e praga do solo ocorre um deslocamento à esquerda, na direção do comprimento de onda do vermelho (700 a $713 \mathrm{~nm})$. Observa-se ainda que os menores valores de REP foram obtidos para a cana-de-açúcar infectada por nematoides, em comprimentos de onda próximos a região do vermelho, na posição de $702 \mathrm{~nm}$. O diagrama mostra também que a amostra coletada em plantas afetadas pelo Migdolus fryanus definiu menor variabilidade nos valores da REP. Na tabela 1 são apresentadas as estatísticas descritivas (média e desvio padrão) calculadas para os valores de REP de cada uma das ocorrências, assim como os comprimentos de onda mínimo e máximo de REP.

Tabela 1: Comprimento de onda médio do REP e respectivos desvios padrão e valores de REP mínimo e máximo obtidos para as ocorrências sadia, infectada por nematoides e por Migdolus fryanus.

\begin{tabular}{c|c|c|c}
\hline & Sadia & Nematóides & M. fryanus \\
\hline Média do REP $(\mathrm{nm})$ & 718,9 & 709,4 & 711,121 \\
\hline Desvio padrão & 3,107 & 3,100 & 0,016 \\
\hline REP mínimo observado $(\mathrm{nm})$ & 714 & 702 & 711 \\
\hline REP máximo observado $(\mathrm{nm})$ & 723 & 711 & 714 \\
\hline
\end{tabular}

O comprimento de onda médio da REP calculado a partir dos elementos amostrais adquiridos para plantas sadias foi maior que aquele calculado para os dois tipos de plantas infectadas, as quais definiram médias pouco distintas. O desvio padrão obtido para plantas infectadas por Migdolus fryanus, porém, foi muito inferior do que para as demais categorias, reiterando sua menor dispersão, conforme mostra a Figura 4.

Por meio de análise estatística t-pareado, verificou-se que os valores médios da REP obtidos para os dois tipos de infecção não foram significativamente diferentes, indicando a necessidade de parâmetros adicionais para distinguir a cana-de-açúcar infectada por nematoides daquela infectada por Migdolus fryanus. Todavia, esse mesmo indicador mostrou diferença significativa 
entre as médias calculadas para cana-de-açúcar infectada e sadia, evidenciando que a posição da REP discrimina o vegetal sadio do infectado, sem a necessidade de parâmetros adicionais.

Os índices hiperespectrais associados às clorofilas $a$ e $b$, a carotenoides, além do NDVI, MCARI e TCARI, constituíram os parâmetros adicionais usados para avaliar a separação espectral entre os tipos de infecção, já que apresentam, entre outras vantagens, a característica de minimizar o efeito de variações nas condições de iluminação da cena.

As médias calculadas a partir dos valores de cada um dos índices anteriores estão relacionadas na Tabela 2, para cada uma das três ocorrências.

Tabela 2: Valores médios e respectivos desvios padrão (dp) dos índices hiperespectrais definidos para as categorias Sadia, infectada por nematoides e por Migdolus fryanus.

\begin{tabular}{l|l|l|l|l|l|l}
\cline { 2 - 7 } & \multicolumn{2}{l}{ Sadia } & \multicolumn{2}{l|}{ Nematoides } & \multicolumn{2}{l}{ M. fryanus } \\
\cline { 2 - 7 } & média & $\mathrm{dp}$ & media & $\mathrm{dp}$ & média & $\mathrm{dp}$ \\
\hline Clorofila a & 10,14 & 0,945 & 8,478 & 0,116 & 5,109 & 0,744 \\
\hline Clorofila b & 6,412 & 0,715 & 6,593 & 0,128 & 4,221 & 0,407 \\
\hline Carotenoides & 13,31 & 1,199 & 9,647 & 0,305 & 7,740 & 2,160 \\
\hline NDVI & 0,799 & 0,013 & 0,773 & 0,009 & 0,633 & 0,053 \\
\hline MCARI & 0,121 & 0,005 & 0,118 & 0,034 & 0,087 & 0,053 \\
\hline TCARI & 0,366 & 0,020 & 0,363 & 0,008 & 0,262 & 0,048 \\
\hline
\end{tabular}

Diferente do comportamento médio observado para a REP, os índices hiperespectrais resultaram em diferenças maiores entre as médias calculadas para nematoides e Migdolus fryanus, indicando seu potencial para discriminar entre parasita e praga. Os índices de Clorofila $b$, NDVI, MCARI e TCARI definiram médias similares para plantas sadias e infectadas por nematoides, porém os valores médios dos índices de Clorofila $a$ e Carotenoides revelaram maior discrepância entre as três ocorrências.

Para expressar graficamente o potencial desses índices em discriminar as ocorrências de interesse, foram elaborados os diagramas boxplots mostrados na Figura 5, os quais indicam os valores médios e intervalo de variação obtidos para os índices de clorofila a (Equação 2), clorofila b (Equação 3) e carotenoides (Equação 4), bem como na Figura 6, que ilustra os boxplots gerados para o NDVI (Equação 5), MCARI (Equação 6) e TCARI (Equação 7).

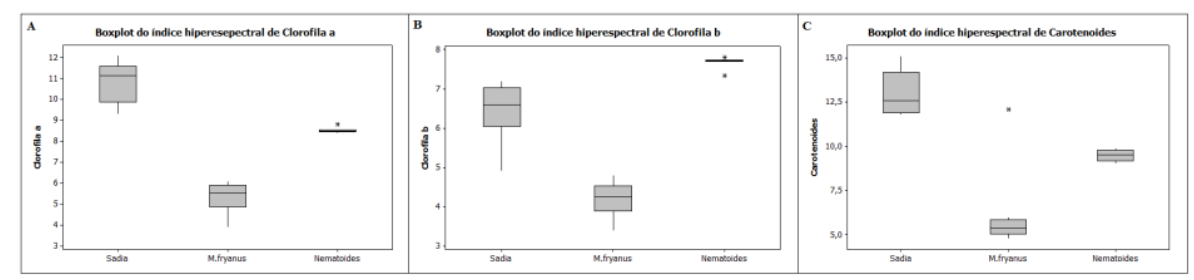

Figura 5: Boxplots dos índices hiperespectrais Clorofila $a$ (a), Clorofila $b$ (b) e Carotenoides (c) gerados a partir das médias e intervalos de variação obtida das amostras de vegetação sadia, infectada por nematoides e por Mygdolus fryanus. 


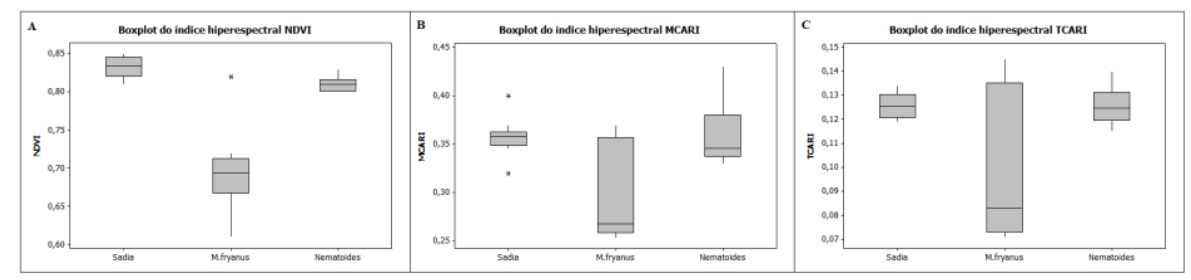

Figura 6: Boxplots dos índices hiperespectrais NDVI (a); MCARI (b); TCARI (c), gerados a partir das médias e intervalos de variação obtida das amostras de vegetação sadia, infectada por nematoides e por Mygdolus fryanus.

Os três índices sensíveis a pigmentos mostrados na Figura 5 definiram boxplots isolados para os diferentes tipos de ocorrência, mas Clorofila $a$ e Carotenoides indicaram maior separação entre a vegetação infectada por nematoides e Migdolus fryanus. Assim, a informação mais significativa revelada pela Figura 5 é a definição de boxplots isolados para os diferentes tipos de ocorrência e a possibilidade de alguns dos índices discriminarem a vegetação sadia e entre os dois tipos de infecção.

Na Figura 6 percebe-se a formação dos boxplots das três ocorrências em intervalos bem definidos de valores de índices hiperespectrais: altos valores para NDVI (de 0,65 a 0,85); intermediários pra MCARI (de 0,25 a 0,40) e baixos para TCARI $(00,7$ a 0,14), porém nenhum desses índices sugere discriminação entre as três ocorrências. O NDVI resultou nos menores valores para a vegetação parasitada pela larva do besouro Migdolus fryanus, apontando para maior comprometimento da cultura quando submetida a esse tipo de infecção, mas apresenta alguma sobreposição entre os boxplots gerados para cana-de-açúcar sadia e infectados por nematoides. Os índices hiperespectrais TCARI e MCARI, além de não mostrarem potencial para separar as três ocorrências (devido à perceptível sobreposição entre os boxplots gerados), indicaram as maiores variabilidades nas medidas tomadas na vegetação parasitada, principalmente por Migdolus fryanus. Isso revela que a incorporação da resposta espectral do verde-visível $(550 \mathrm{~nm})$ na formulação desses dois últimos índices espectrais, não favoreceu a discriminação das amostras infectadas pela praga e parasita.

Assim como a REP, os índices hiperespectrais NDVI, MCARI e TCARI não permitiram separar entre os tipos de infecção, mesmo considerando a quase simultaneidade na tomada das medidas de FRHC na vegetação sadia e a infectada, as quais foram tomadas acima do dossel da cana-deaçúcar. Além disso, a formulação desses índices remete a minimização do efeito das condições de iluminação (Ponzoni et al., 2015). Essas métricas, porém, assim como o índice de clorofila $b$, possibilitaram discriminar apenas entre plantas sadias e infectadas.

No estudo realizado, os índices sensíveis aos pigmentos clorofila $a$ e carotenoides discriminaram inclusive entre as plantas infectadas por nematoides e Migdolus fryanus, o que é explicado pelo fato dos pigmentos foliares fornecerem informações sobre o estado fisiológico das plantas. $\mathrm{O}$ conteúdo de carotenoides, especificamente, está relacionado com estresse da planta, além da capacidade fotossintética (Hernandez-Clemente et al., 2012).

Especificamente no que se refere ao desenvolvimento metodológico adotado, deve-se enfatizar o cuidado tomado na aquisição dos dados in situ, a qual foi orientada por protocolos consagrados, a fim de minimizar o efeito de fatores externos na aquisição das medidas. Além disso, a discriminação espectral entre os diferentes tipos de ocorrência incluiu a análise do resultado da aplicação de índices espectrais que, por definição, minimizam a influência das condições de iluminação. 
Finalmente, é necessário destacar que as áreas infectadas por parasitas e pragas do solo definem um padrão espacial (como pode ser verificado na cena RapidEye, incluída na Figura 1) que permite sua detecção por sensores remotos instalados em plataformas aéreas e mesmo orbitais, quando em estágios mais avançados de infecção. Nesse sentido, uma indicação dos intervalos e índices espectrais que favorecem a detecção de áreas infectadas pode contribuir para dimensionar a área comprometida e na definição de práticas de manejo. No presente caso, a Figura 6 mostra que o NDVI discriminou melhor as ocorrências e que a inclusão de uma banda adicional (em $550 \mathrm{~nm}$ ) não aumentou a separação entre elas.

\section{CONCLUSÕES}

1 - As curvas de reflectância obtidas por radiometria de campo para cana-de-açúcar sadia e infectada por nematoides e Migdolus fryanus definiram diferenças apenas em magnitude. O espectro de plantas sadias e parasitadas por nematoides mostraram a banda de absorção no vermelho, porém plantas infectadas pela larva do besouro indicaram valores médios de FRHC maiores nessa região espectral. No infravermelho próximo, plantas infectadas pela praga e parasita mantiveram patamares similares de reflectância, consideravelmente menores que as sadias.

2 - A determinação do comprimento de onda da posição da borda do vermelho limítrofe (REP) permitiu discriminar plantas sadias e infectadas. No entanto, os valores médios da REP obtidos para as plantas submetidas aos dois tipos de infecção não foram significativamente diferentes.

3 - As métricas extraídas dos dados hiperespectrais NDVI, MCARI e TCARI, assim como o índice de clorofila $b$ também viabilizaram a separação entre plantas sadias e infectadas, mas não entre os tipos de infecção. Essa constatação reafirma as diferenças significativas entre as características espectrais da vegetação sadia e infectada.

4 - Confirmando a condição de que o conteúdo de pigmentos foliares está associado ao estado fisiológico da planta, os índices sensíveis à clorofila $a$ e carotenoides discriminaram as três ocorrências estudadas, inclusive entre as plantas infectadas por nematoides e Migdolus fryanus. A discriminação mais eficiente foi obtida com o índice de carotenoides, considerando que seu conteúdo está relacionado com estresse da planta, além da capacidade fotossintética.

\section{AGRADECIMENTOS}

À empresa sucroalcooleira Umoe Bioenergy de Sandovalina, SP pela disponibilidade das áreas de pesquisa e de técnicos especializados para o acompanhamento de campo. Ao Programa de Pós-graduação em Ciências Cartográficas e Departamento de Cartografia da UNESP pelo auxílio financeiro e por disponibilizar os instrumentos utilizados na pesquisa. Ao Conselho Nacional de Desenvolvimento Científico e Tecnológico (CNPq) pela concessão de bolsa. 


\section{REFERÊNCIAS BIBLIOGRÁFICAS}

Abdel-Rahman, Elfatih, and Ahmed, Fethi B. The application of remote sensing techniques to sugarcane (Saccharum spp. hybrid) production: a review of the literature. International Journal Of Remote Sensing. Bristol, Pa, Usa, p. 3753-3767. jul. 2008.

Apan, Armando; Held, Alex; Phinn, Stuart; Markley, John. Detecting sugarcane 'orange rust' disease using EO-1 Hyperion hyperspectral imagery. International Journal of Remote Sensing, 25. pp. 489-498. 2005.

Bégué, Agnes; Lebourgeois, Valentine; Bappel, Eric; Todoroff, Pierre; Pellegrino, Anne. Spatiotemporal variability of sugarcane fields and recommendations for yield forecast using NDVI. International Journal of Remote Sensing, 31 (20), pp.5391-5407, 2010.

Blackbum, Allan G. Quantifying chlorophylls and carotenoids at leaf and canopy scales: an evaluation of some hyperspectral approaches. Remote Sensing of Environment, v.66, n.3, p.273-285. 1998.

Carter, Gregory A. Primary and Secondary effects of the water content of the spectral reflectance of leaves. American Journal of Botany, v.74, n.7, p.916-924, 1993.

Cho, Moses A., and Skidmore, Andrew K. A new technique for extracting the red edge position from hyperspectral data: The linear extrapolation method. Remote Sensing of Environment, v.101, p.181-193, 2006.

Corbani, Renato Zampparoli. Estudo do extrato pirolenhoso biopirol® no manejo de nematóides em canade-açúcar, olericolas e citros em diferentes ambientes. 53p. Tese (Doutorado em Agronomia). Faculdade de Ciências Agrárias e Veterinárias, Universidade Estadual Paulista, Jaboticabal, 2008.

Daughtry, C. S. T.; Walthall, C. L.; Kim, M. S.; Brown de Colston, E.; Mcmurtrey, J. E. Estimating corn leaf chlorophyll concentration from leaf and canopy reflectance. Remote Sensing of Environment. v.74, n.2, p. 229-239, 2000.

GALVÃO, Lênio Soares; FORMAGGIO, Antônio Roberto; TISOT, Daniela Arnold. Discrimination of sugarcane varieties in Southeastern Brazil with EO-1 Hyperion data. Remote Sensing Of Environment, Usa, v. 94, n. 4, p.523-534, 28 fev. 2005.

Gonçalves, Renata R. V.; Zullo JR, Jurandir, Romani, Luciana A. S.; Nascimento, Cristina R.; Traina, Agma J. M. Analysis of NDVI time series using cross-correlation and forecasting methods for monitoring sugarcane fields in Brazil. International Journal of Remote Sensing, v.33, n.15, p.4653-4672, 2012.

Grisham, Michael P.; Johnson, Richard M.; Zimba, Paul V. Detecting sugarcane yellow leaf virus infection in asymptomatic leaves with hyperspectral remote sensing and associated leaf pigment changes. Journal of Virological Methods. v.167, p.140-145, 2010.

Haboudane, Driss; Miller, John R.; Tremblay, Nicolas; Zarco-Tejada, Pablo J.; Dextraze, Louise. Integrated narrow-band vegetation indices for prediction of crop chlorophyll content for application to precision agriculture. Remote Sensing of Environment, v.81, p.416-426, 2002. 
Hernendez-Clemente, Rócio; Cerrillo, Rafael M. N, Tejeda, Pablo. J. Z. Carotenoid content estimation in a heterogeneous conifer forest using narrow-band indices and PROSPECT + DART simulation. Remote Sensing of Environment, v.127, p.298-315, 2012.

Jensen, John R. Sensoriamento remoto do ambiente: uma perspectiva em recursos terrestres. São José dos Campos: Parêntese. 672p, 2009.

Ju, Chang Hua; Tian, Yong Chao.; Yao, Xia; Cao, Wei Xing.; ZHU, Y.; HANNAWAY, D. Estimating Leaf Chlorophyll Content Using Red Edge. Pedosphere, v.20, n.5, p.633-644, 2010.

Kimes, Daniel S.; Smith, Jack A.; Ranson, Kenneth. J. Interpreting vegetation reflectance measurements as a function of solar zenith angle. NASA Technical Memorandum 8030. Greenbelt, Maryland: Goddard Space Flight, p.3-36, 1979.

Liu, Jiangui; Pattey, Elizabeth.; Miller, John. R.; Mcnairn, Heather; Smith, Anne; Hu, Baoxin. Estimating crop stresses, above ground dry biomass and yield of corn using multi-temporal optical data combined with a radiation use efficiency model. Remote Sensing of Environment, v.114, p.1167-177, 2010.

Martonchick, John V.; Bruegge, Carol J.; Strahler Alan H. A review of reflectance nomenclature used in remote sensing. Remote Sensing Review, v.19, p.9-20, 2009.

Milton, Edward J.; Schaepman, Michael E.; Aanderson, Karen; Kneubühler, Mathias; Fox, Nigel. Progress in field spectroscopy. Remote Sensing of Environment, v.113, p.92-109, 2009.

Omarjee, Jehhan, Balandreau Jacques., Spaull, Vaughan W.; Cadet, Patrice. Relationships between Burkholderia populations and plant parasitic nematodes in sugarcane. Applied Soil Ecology, v.39, n.1, p1-14, 2008.

Ponzoni, Flávio J.; Shimabukuro, Yosio E.; Kuplich, Tatiana M. Sensoriamento Remoto no Estudo da Vegetação. São José dos Campos: Parêntese, 160p, 2015.

RAO, Rama N. Development of a crop specific spectral library and discrimination of various agricultural crop varieties using hyperspectral imagery. International Journal of Remote Sensing, v.29, n.1, p.131-144, 2008.

ROUSE, J. W.; HAAS, R. H.; SCHELL, J. A.; DEERING, D. W. Monitoring vegetation systems in the Great Plains with ERTS. In: Earth Resources Technology Satellite-1 Symposium, 3, 1973, Washington, DC. Proceedings... Washington, DC: NASA, 1973. p. 309-317.

Rudorff, Bernardo F. T.; Aguiar, Daniel A.; Silva, Wagner F.; Sugawara, Lucina M.; Goltz, Elizabeth; Aulicino, Tânia L. I. N.; Carvalho, Magog A.; Brandão, Daniela; Arenas-Toledo, John M. Uso de imagens de satélites de sensoriamento remoto para mapear a área cultivada com cana-de-açúcar no estado de São Paulo - safra 2009/10. São José dos Campos: INPE, 46 p., 2010.

Scheapman-Strub, Gabriela; Scheapman, Michael E.; Painter, Thomas. H.; Dangel, Stefan.; Martonchik John V.; Reflectance Quantities in Optical Remote Sensing - Definitions and Case Studies. Remote Sensing of Environment, v.103, n.1, p.27-42, 2006.

Recebido em março de 2015.

Aceito em agosto de 2015. 